\title{
The Furin Cytoplasmic Domain Is Localized to the trans-Golgi Network of Yeast
}

\author{
Alison J. Farrell \\ Biological Sciences, Central Washington University, 400 E. University Way \\ Ellensburg, WA 98926, USA \\ Tel: 425-820-2685 E-mail: alison101@comcast.net \\ Katherine A. Keltner \\ Biological Sciences, Central Washington University, 400 E. University Way \\ Ellensburg, WA 98926, USA
}

Tel: 509-373-0409 E-mail: Katherine_A_Keltner@rl.gov

Kathryn L. Bruce

Biological \& Environmental Sciences, CBX081, Georgia College \& State University

Milledgeville, Georgia, 31061, USA

Tel: 706-474-1200Ｅ-mail: klynbruce@gatech.edu

Jessica L. Snell

Biological \& Environmental Sciences, CBX081, Georgia College \& State University

Milledgeville, Georgia, 31061, USA

Tel: 770-365-2018Ｅ-mail: jessica.1.snell@gmail.com

Mark F. Law

Biological \& Environmental Sciences, CBX081, Georgia College \& State University

Milledgeville, Georgia, 31061, USA

E-mail: markf_law@yahoo.com

Matthew C. Furgerson

Biological \& Environmental Sciences, CBX081, Georgia College \& State University

Milledgeville, Georgia, 31061, USA

E-mail: mfurgerson@bmb.uga.edu

David B. Nix

Biological \& Environmental Sciences, CBX081, Georgia College \& State University

Milledgeville, Georgia, 31061, USA

Tel: 404-395-2349 E-mail: dnix@bmb.uga.edu

Michael L. Gleason (Corresponding author)

Biological \& Environmental Sciences, CBX081, Georgia College \& State University

Milledgeville, Georgia, 31061, USA

Tel: 478-445-0813 E-mail: mike.gleason@gcsu.edu 
Received: August 16, 2010 Accepted: August 31, 2010 doi:10.5539/ijb.v3n3p3

This research was supported by an NIH AREA grant 1R15GM51089-01 and faculty research grants from the $C W U$ and GCSU Foundations.

\begin{abstract}
Kex2p and furin are membrane-bound proteases localized to the trans-Golgi network (TGN) of yeast and animal cells. While their N-terminal lumenal and catalytic domains are highly homologous, they do not share common sorting signals in their C-terminal cytoplasmic tails. To study furin's localization in yeast we created KFp chimeras with the $\mathrm{N}$-terminal regions of Kex $2 p$ and the C-terminal regions of human furin. Our mating, biochemical, and immunofluorescence studies of the chimeras in yeast have demonstrated sorting consistent with furin's trafficking patterns in human cells. Next, to see if furin sorting signals are recognized by yeast sorting machinery, key signals were mutated. For two mutagenized chimeras (KF5p/6p), mating activity was abolished, indicating that the enzyme no longer resides in the yeast TGN. This is surprising since the furin sorting signal that was altered normally binds a sorting protein (PACS-1) not known to exist in yeast.
\end{abstract}

Keywords: Furin, Kex2p, Protease, PACS-1, Trans-Golgi network, Endosome, Sorting, Localization

\title{
1. Introduction
}

\subsection{Kex2p and furin are homologous proteases localized to the trans-Golgi network}

Conserved cellular machinery is a common feature of eukaryotes. An interesting example of this is found for two proteases, furin, common to animal cells, and Kex2p, found in Saccharomyces cerevisiae and other yeast. Besides sharing significant structural homology, these two serine proteases are also strikingly similar in catalytic specificity and cellular function. Both are type I membrane-bound proteins, principally localized to the trans-Golgi network (TGN), that proteolytically process and activate other proproteins passing through the secretory pathway (Fuller et al., 1989b; Takahashi et al., 1995; van den Ouweland et al., 1990).

Kex2p was the first of the two proteases to be characterized genetically and biochemically (Fuller et al., 1989a). Soon followed the discovery of a family of related membrane-bound subtilisin-like serine proteases that began with furin (Fuller et al., 1989b) and then led to seven other animal paralogs (Rockwell et al., 2002; Thomas, 2002). Kex $2 p$ and furin are both endoproteases that hydrolyze the carboxyl-side peptide bond of dibasic amino acid sequences. Kex2p cleaves pro-killer toxin, mating factor- $\alpha$ polypeptides 1 and 2, and Keilp (Julius et al., 1984; Sato et al., 2009; Wickner \& Leibowitz, 1976), while over 40 different animal proproteins and animal pathogen proproteins are thought to be cleaved and activated by furin (Thomas, 2002). Both proteases are extensively modified in the endoplasmic reticulum (ER) and Golgi by signal peptidase cleavage, glycosylation, and their own autohydrolysis of the N-terminal pro-region that delivers each as a proteolytically active enzyme to the TGN of their respective cells (Thomas, 2002). Structurally, the domains of the furin and Kex2p enzymes show a high-degree of conservation along their entire length, but significant sequence alignment and amino acid identity is only present in their N-terminal domains (Fig. 1). Conversely the final quarter of each protein (their C-terminal regions) show structural homology, but no significant sequence homology.

\subsection{Maintenance of TGN proteins}

A major question in membrane trafficking is how resident TGN proteins are maintained against the flow of Golgi contents passing beyond this compartment (Bonifacino \& Glick, 2004; Bonifacino \& Rojas, 2006). Early studies revealed that retrotransport from the endosomal compartments is the major means by which TGN proteins are maintained in this compartment, and although less understood, TGN retention plays a role as well. This was first proposed for Kex2p based largely on the observations that it is rapidly lost to the vacuole when its cytoplasmic tail is truncated and by pulse-chase experiments that revealed native Kex2p experiences increasing glycosylation with longer chase times (Fuller et al., 1989b). It was later shown that MNN1-dependent mannosylation of N-linked core oligosaccharides on membrane proteins occurs with increased residency within the late Golgi of yeast (Ballou et al., 1990). Other studies by Brickner \& Fuller (1997) discovered retrieval and retention signals in the cytoplasmic tail of Kex2p. Laboratories studying the localization of furin, as reviewed by Thomas (2002), confirmed that rapid cycling of furin follows a similar pattern, and that it too contains sorting signals in its cytoplasmic tail. Thus, retrotransport to the TGN is thought to maintain these resident proteins at a high steady-state in the TGN, while their presence in distal compartments is, by comparison, more transitory (Bonifacino \& Rojas, 2006). While descriptions of endosomal compartments are varied and complex, it is 
generally held that endocytic vesicles fuse with early endosomes (EE) that then mature into late endosomes, also known as the pre-vacuolar compartment (PVC) in yeast. Endosomes exist in equilibrium with the cell surface and the TGN as vesicles transport membrane and contents between them, and by maturation of the late endosome into the lysosome in animal cells, or its analog in yeast, the vacuole.

\subsection{Maintenance of Kex2p in the yeast TGN}

Retention and retrieval of Kex2p and DPAP A, a related proprotein protease in yeast, involves two well-studied sequence signals within the cytoplasmic tails of these enzymes, the trans-Golgi localization signal-1 (TLS-1) and TLS-2 (Brickner \& Fuller, 1997; Bryant \& Stevens, 1997; Wilcox et al., 1992). Mutational studies strongly implied that a tyrosine in TLS1 $\left(\mathrm{Y}^{713} \mathrm{XF}\right)$ in Kex2p is critical for retrieval of the native Kex2p from an endosomal compartment, as yeast expressing fully active $\mathrm{A}^{713}$-enzyme rapidly lose the protease to the vacuole (Wilcox et al., 1992). Downstream of TLS1 is TLS2, which under Soilp suppression of the $\mathrm{Y}^{713} \rightarrow$ A mutation delays the exit of Kex2p from the TGN (Brickner \& Fuller, 1997; Redding et al., 1996a).

Many sorting proteins are involved with the exit of Kex $2 p$ from the yeast TGN. Studies of $c h c 1$ clathrin heavy chain mutants and $c h c 1$ secl mutants also blocked for secretion demonstrated that any Kex $2 p$ that is lost to the cell surface is rapidly internalized by a non-clathrin mediated endocytosis (Payne \& Schekman, 1989; Redding et al., 1996b). This and other work established that Kex2p's main route of exit from the TGN is to the PVC. This "direct" pathway is also taken by the well-studied yeast receptor Vps10p (Bowers \& Stevens, 2005). However, defects in the phosphoinositide phosphatase, Inp53p, were found to correlate with a slow delivery of Kex2p and Ste13p to the PVC, which indicated that these proteins may also be arriving at the PVC by an "indirect" route from the TGN (Ha et al., 2001), presumably via the early endosome (EE). This was confirmed when the Fuller lab determined that Kex2p partitions its forward transport from the TGN by both the direct and indirect pathways (Sipos et al., 2004). Vesicular transport of Kex2p to the PVC is known to require clathrin, adaptor proteins Ggalp/2p, and the dynamin, Vps1p, with fusion of the Kex2p-containing vesicle at the PVC requiring the t-SNARE Pep12p (Abazeed \& Fuller, 2008; Ha et al., 2001). The proteins involved in the forward transport of Kex2p from the TGN to the EE, include clathrin, adaptor protein AP-1, and Inp53p (Sipos et al., 2004; Abazeed \& Fuller, 2008). Kex2p delivered by the "indirect" path from the EE will eventually reside in the PVC as Soi3p facilitates the acidification of the EE compartment leading to its maturation into a late endosome (Sipos et al., 2004).

Retrotransport pathways from endosomes to the TGN often involve a retromer complex (Seaman, 2005). This process was initially discovered in studies that examined how the membrane-based receptor Vps10p delivers procarboxypeptidase $\mathrm{Y}$ (proCPY) to the yeast $\mathrm{PVC}$ and is then retrieved to the yeast TGN by a complex of retromer proteins (Seaman et al., 1998). Since then, animal cell homologs, called sorting nexins (SNXs), have been shown to act similarly (Verges, 2007). Indeed, Kex2p is known to require an ortholog of the sorting nexin SNX3, known as Grd19p, for its retrieval from the PVC (Voos and Stevens, 1998). Likewise, Mvp1p, an ortholog of human sorting nexin SNX8, has been implicated in a retromer-based Kex2p retrieval from the PVC (Ekena \& Stevens, 1995). Little is directly known about the retrieval of Kex2p from the EE membrane, except that it does require Tlg1p, Tlg2p and Vtilp to form a t-SNARE complex known to mediate fusion of endosomal vesicles with the yeast TGN (Bonifacino \& Rojas, 2006).

\subsection{Maintenance of furin in the animal cell TGN}

The cellular localization of furin in human and animal cells is decidedly within the TGN, and nearly all of its suspected proproteins are activated in this compartment (Molloy et al., 1999; Thomas, 2002). However, a small fraction of furin transits through the cell surface and endosomal compartments where some opportunistic animal pathogen proproteins are activated by proteolysis (Molloy et al., 1994; Bosshart et al., 1994). Furin's transport to the cell surface may be explained by many observations. It has been noted that clathrin and adaptor proteins AP-1 or AP-4 may participate in a small fraction of it being constitutively transported from the TGN to the cell surface (Schäfer et al., 1995; Teuchert et al., 1999a; Rohn et al., 2000). It has also been speculated that, in the absence of sorting signals, single-pass proteins with a somewhat longer TMD, like furin's compared to that of Kex2p, are more likely to be lost from the Golgi and then retained at the cell surface within lipid rafts (Bosshart et al., 1994; Lingwood \& Simons, 2010; Rayner \& Pelham, 1997; Schäfer et al., 1995). Another proposed pathway to the cell surface involves furin's inclusion in regulated secretory granules (Dittié et al., 1997). The strongest evidence supports a path for furin to the cell surface via an endosomal compartment (Thomas, 2002) or compartments (Molloy et al., 1999) that may parallel Kex2p's presumed pathway for arrival at the cell surface in mutant yeast (Payne \& Schekman, 1989). For example, AP-1 is thought to bind to the furin sorting signal $\mathrm{Y}^{759} \mathrm{KGL}$ (Fig. 2, amino acids in green) to facilitate vesicular budding of furin from the TGN to an endosomal or 
secretory compartment (Crump et al., 2001). While most the furin is retrotransported to the TGN, a small portion of it reaches the cell surface via a sorting signal-directed EE to cell surface step (Thomas, 2002).

Regardless of how furin gets to the animal cell surface, it is ultimately returned to an endosomal pool of the protease by the sorting signal motif, $\mathrm{Y}^{759} \mathrm{KGL}$, which interacts with AP-2 to enable clathrin-mediated endocytosis (Teuchert et al., 1999b). Thomas (2002) has proposed a shuttling mechanism in which furin is directly transported to an endosomal compartment, that is then followed by clathrin and AP1 (or AP4) retrotransport to the TGN. Key to furin's retrotransport is a phosphophorylated acidic cluster (AC) in its cytoplasmic tail (Fig. 2, amino acids in red), that mutational studies have defined and further studies have indicated must be bound by the phosphofurin acidic cluster sorting protein-1 (PACS-1) during its retrotransport (Schäfer, et al., 1995; Takahashi, et al., 1995; Voorhees et al., 1995; Wan et al., 1998). AC is a cluster of acidic amino acids and two closely-spaced serines that are phosphorylated by casein kinase II. Additionally, adaptor proteins and clathrin are thought to interact with furin through a PACS-1 intermediate.

\subsection{Study of furin sorting signals in yeast}

In this paper, we report our investigations that ask if $\mathrm{C}$-terminal furin sorting signals in yeast are sufficient to localize Kex $2 p$ to the TGN? To study this, we chimerically-fused the N-terminal pre-pro-enzymatic and regulatory domains of the Kex2p coding regions of the yeast $K E X 2$ gene $(\mathrm{K})$ to the $\mathrm{C}$-terminal regions of furin encoded by the human fur cDNA (F) to create chimeric constructs KFJp and KF2p (see Figs. 1 and 2). Intriguingly, we initially observed that the chimeric enzyme (KFp) is well-retained in the yeast TGN by several measures, and our findings support a trafficking pattern in yeast that is typical of furin in animal cells. This led us to wonder whether any of the human furin sorting signals are being recognized by conserved sorting machinery native to the yeast cell, and to our surprise we report mating results for site-specific mutations to the $\mathrm{AC}$ that are consistent with a hypothetical PACS-1-like retrieval of the chimeric enzyme from yeast endosomes to the TGN.

\section{Experimental Procedures}

All standard reagents and culture media used in these experiments were purchased from Sigma-Aldrich, Fisher Scientific, BIO-RAD, or VWR Scientific.

\subsection{Bacterial and Yeast Strains and their Growth}

Standard bacterial E. coli strains used for molecular cloning were derivatives of DH5 $\alpha$ or strains purchased as commercially competent cells such as XL1-Blue or XL10-Gold from Stratagene/Agilent Technologies. Growth of E. coli and preparation of competent cells were as described (Sambrook et al., 2000).

The two principle yeast strains used in these studies were BFY106-4D (Pep4 ${ }^{+}$) and CB017 (Pep4 ${ }^{-}$). These strains were derived by the Fuller lab from a parental W303-1A yeast strain (R. Rothstein, Columbia University, NY) as described by (Wilcox et al., 1992). Both strains are MATa and contain a deletion of the gene for Kex2p (kex2A). The full genotype for each of the two strains is as follows:

$$
\begin{aligned}
& \text { BFY106-4D (Pep4 }{ }^{+} \text {): MAT } \alpha \text { kex2A::HIS3 can1-100 ade2-1 his3-11,-15 leu2-3,-112 trp1-1 ura3-1 } \\
& \text { CB017 (Pep4-): MATa kex2A::TRP1 pep4::HIS3 prb1::hisG prc2::hisG can1-100 ade2-1 his3-11,-15 } \\
& \text { leu2-3,-112 trp1-1 ura3-1 }
\end{aligned}
$$

As indicated above, BFY106-4D is phenotypically wild-type for vacuolar proteases (Pep4 ${ }^{+}$), while CB017 is significantly impaired for proteolytic turnover for proteins $\left(\mathrm{Pep}^{-}\right)$delivered to its vacuoles due to the deletion of three key vacuolar protease genes: PEP4, PRB1, and PRB2. Two strains used for the mating test were DC14 MATa his 1 and DC17 MATa his 1 which originated in the J. Thorner lab (U.C. Berkeley). Standard growth and maintenance of strains was on rich media (e.g., YPD) and synthetic drop-out media (e.g., SDC-ura) as described by (Sherman, 2002). The addition of $2 \%$ D-galactose was used to induce transcription driven by a GAL1 promoter. For some experiments yeast strains were grown by in liquid broths by shaking at $30^{\circ} \mathrm{C}$ until cell density reached mid-log or late-log phase $\left(\mathrm{OD}_{600} 0.5\right.$ or 1.0$)$.

\subsection{Plasmids, Cloning and Mutagenesis}

The original chimeric construct, $K F J$ (Fig. 2) was constructed from genomic KEX2 DNA and human fur cDNA by J. Burchard in the Fuller lab, and was then placed into pBM258 plasmid to create pBM258-KFJ used in some of our studies. pBM258 is a standard YCp50 plasmid containing the GAL10,1 promoter region (Davis, 1989; Johnston \& Davis, 1984). Using pBM258-KX22 described by (Fuller et al., 1989a) and human fur cDNA (Open Biosystems), we constructed a new chimera with eleven codons removed from the 5'-end of the C-terminal coding region, we called KF2 (Fig. 2). Enzymes required for cloning were obtained from New England Biolabs. 
We then placed $K F 2$ under the control of the $G A L 1$ promoter in pBM258, creating pBM258-KF2. From pBM258-KF2, we created site-specific mutants in the cytoplasmic tail of furin called KF3, KF5, and KF6 (Fig. 2). Mutagenesis was done using a QuikChange XL Site-Directed Mutagenesis Kit (Stratagene/Agilent Technologies). The plasmids pBM258-KX22 and pCW-KX20 (Wilcox et al., 1992) are nearly equivalent constructs used in our experiments that both express the positive control, native Kex2p, via a GAL1 promoter. The plasmid pBM258 is as described, and since it contains no structural gene for $K E X 2$, it was also used as a negative control vector for some studies.

\subsection{Kex2p specific Proteolytic Assay of Intact Cells}

Non-saturating proteolysis reactions were conducted by a one-hour assay at $30^{\circ} \mathrm{C}$ on Cbz-YKR-pNA (Bachem) at $405 \mathrm{~nm}$ as described (Achstetter et al., 1984). Additional Kex2p-specific assay conditions were required (Fuller et al., 1989a) and necessitated the use of the following protease inhibitors to eliminate the action of competing proteases: 1- $\mu \mathrm{M}$ E-64, 25- $\mu \mathrm{M}$ Pepstatin A, 100- $\mu \mathrm{M}$ TPCK, 500- $\mu \mathrm{M}$ PMSF, and 100- $\mu \mathrm{M}$ TLCK. Kex2p-specific activity of intact mid-log cells was determined in $50-\mathrm{mM}$ Tris-HCl, $\mathrm{pH} 7.5$ buffer containing protease inhibitors as above and as described (Wilcox et al., 1992). Lysis of intact yeast was accomplished by a $3 \mathrm{X}$ freeze/thaw lysis with liquid nitrogen frozen cells thawed on ice in the above buffer which included $1 \%$ Na-deoxycholate and $1 \%$ Triton-X-100.

\subsection{Immunofluorescence Microscopy}

Mid-log yeast Pep4 $4^{+}$or Pep4 ${ }^{-}$cells expressing: no enzyme (pBM258), Kex2p (pCW-KX20), or KFJp (pBM258-KFJ) were prepared for microscopy by fixation in buffered $1 \%$ formaldehyde, followed by spheroplasting with Zymolyase and then applied to poly-lysine-coated slides as described (Wilcox et al., 1992). Acetone-permeabilized cells were then pre-treated with pre-immune rabbit serum and then labeled with $\alpha$-ssKex $2 p$ rabbit polyclonal antibody sandwiched to goat- $\alpha$-rabbit IgG and rabbit- $\alpha$-goat-FITC. The antigen used to raise the immune serum, ssKex $2 p$, is a secreted soluble version of Kex $2 p$ missing the spacer-TMD-tail region C-terminal domains (Brenner \& Fuller, 1992), hence it binds to the enzyme moiety of either Kex2p or chimera. The cellular location of Kex2p or chimera was then determined by indirect immunofluorescence on a Leica epifluorescence microscope also capable of differential interference contrast (DIC) microscopy.

From photos of prepared slides, two investigators individually counted the occurrences in which cells exhibited strong fluorescence in punctate structures (Golgi) and halos (cell surface) and, in rare instances, when fluorescence coincided with a vacuole visible by DIC. The presence of multiple labeled structures in a cell (e.g., several punctate structures) was counted as a single occurrence within the cell. The counts of the two investigators were averaged. Cells that exhibited fluorescent labeling of the cell surface (halos) were nearly always (97\% of the time) accompanied by punctate fluorescent staining typical of the Golgi. Thus to simplify their scoring, all cells showing surface fluorescence were counted simply as "+ cell surface" in our data.

\subsection{Mating Assays}

Matings of MAT $\alpha$ cells with testing strains DC14 and DC17 were as described (Redding et al., 1996; Sprague, 1991; Wilcox et al., 1992). Briefly, the expression of Kex2p in the TGN is required for $\alpha$-mating factor production. MATa cells that have mated with MATa DC14 cells will grow as prototrophic diploids on minimal media (SD or SGal), whereas the auxotrophic haploid cells will not. As expected, a consistent lack of mating with DC17 (a MATa strain isogenic to DC14) was regularly demonstrated. All cells grow on YPD, so the fraction of $M A T \alpha$ cells capable of mating can be determined in qualitative patch assays or quantitatively. In quantitative tests MATa cells and DC14 cells were grown in broth to mid to late-log and then adjusted in concentration so that $1.0 \times 10^{6}$ cells $/ \mathrm{ml}$ of MAT $\alpha$ tester cells were mated to $1.0 \times 10^{7}$ cells $/ \mathrm{ml}$ of DC14. For mating, cells were either serially diluted in titer plates and then replicated onto minimal or rich media using a pin-replicating tool or mated in microfuge tubes and then spread uniformly over the surface of a plate. All plates were grown for 2-3 days at $30^{\circ} \mathrm{C}$ before the data were recorded.

\section{Results}

\subsection{Preliminary biochemical analysis indicates that the KFp chimera localizes to the TGN and cell-surface}

The steady-state expression of Kex2p and the KFJp chimera were originally examined by J. Burchard in the Fuller lab (M.S. Thesis, Stanford University, 1992). Importantly, her original experiments determined that both proteins were expressed to the same extent using a western blot analysis. Moreover, when the yeast cells were crudely fractionated, and the membrane fractions expressing KFJp and Kex2p compared, it was observed that the bead-beaten lysates expressing the KFJp chimera had the protein distributed between the ultrahigh-speed microsomal 100S fraction (i.e., Golgi) and a low-speed 10S fraction. By comparison essentially all of the Kex2p in control cells was found in the high-speed microsomal fraction indicative of Kex2p's predominant TGN 
localization (Fuller et al., 1989b). The dense cell fraction (10S) obtained by this type of method is consistent with membrane-cell wall fragments (Sipos \& Fuller, 2002).

\subsection{Quantitation of the physical partitioning of the KFp chimera by assay of its enzymatic domain}

In the corresponding author's laboratory we first followed-up on these initial observations by three means. By the direct enzymatic detection of Kex2p activity, we first examined yeast cell membranes that were gently lysed and fractionated on density gradients, and secondly, directly measured the amount of surface Kex2p activity present relative to total Kex2p activity. The localization of the Kex $2 p$ enzymatic domain was assessed indirectly by immunofluorescence microscopy as the third means.

The Kex2p-specific proteolytic activity of membrane fractions from gently lysed yeast cells were separated on Percoll density gradients (Fig. 3A). The relatively larger peak of Kex2p proteolytic activity in the denser fractions for the cells expressing KFJp in comparison to Kex2p confirms the earlier findings obtained by bead-beaten lysis. The assay is quite specific, as most of the proteolytic activity measured in this Kex2p assay is due solely to the activity of Kex $2 p$ enzymatic domain (Wilcox et al., 1992), with only a small fraction of the proteolysis (1-2\%) being due to competing proteases.

Second, the amount of Kex2p-specific proteolytic activity present on the cell surface of living yeast cells expressing KFJp or Kex2p was compared. The technique used was previously employed by the Fuller lab (Wilcox et al., 1992 and Fuller et al., 1989b). The results of intact Pep4 ${ }^{+}$cell studies (Fig. 3B) confirm that a substantial fraction of KFJp chimera was found on the cell surface and that the amount of enzyme detected was significantly larger than assayed for the Kex $2 p$ control $(p<0.04)$.

A third technique provided a visual localization of the enzyme by indirect immunofluorescence using a polyclonal antibody against the Kex2p enzymatic domain common to both Kex2p and KFJp. It is worth noting that these studies are well-controlled by the fact that Kex2p was established as one of the earliest markers for the yeast TGN and that it is still among the most widely-used biochemical markers for this compartment in yeast (Bonifacino \& Rojas, 2006; Fuller et al., 1989a). Data shown in Fig. 4 demonstrate that the Kex2p enzymatic domain antigen lies in the peripherally distributed Golgi of yeast. This characteristic type of yeast Golgi staining is clearly visible as punctate staining near the plasma membrane as seen for both Pep4 ${ }^{+}$cells expressing the Kex2p control (Fig. 4C) and KFJp chimera (Fig 4E). Companion DIC photos (Figs. 4D and 4F, respectively) show the location of vacuoles in fluorescing cells that by comparison are devoid of fluorescence. Notably, as seen in Fig. 4E, the KFJp chimera is also localized to the plasma membrane. This is revealed by the dim fluorescent halos encircling the yeast's cell membrane. Scoring the occurrence of these two features provided statistically valid data $(\mathrm{p}<0.001)$ as we observed that $39 \%$ of the cells expressing the KFJp chimera had a cell-surface distribution in Pep $4^{+}$cells compared to only $3 \%$ in the Kex $2 p$ controls (Table 1). In Pep $4^{-}$yeast, where vacuolar proteolytic turnover of proteins is known to be impaired (Wilcox et al., 1992), our studies demonstrate approximately $60 \%$ of the fluorescently labeled cells expressing KFJp had a cell-surface distribution. By comparing these cell surface distributions of the chimera in Pep4 $4^{+}$and $\mathrm{Pep}^{-}$yeast, we deduced that KFJp can enter endosomes from the cell surface leading to its turnover in the vacuole. Importantly, the data also support that the KFJp chimera resides in the yeast Golgi as nearly all the fluorescing cells had evidence of TGN localization.

\subsection{Mating activity assays localize the enzymatic domain to the TGN}

Our studies have also utilized a unique measure for Kex2p's TGN localization that takes advantage of the enzyme's principal value to the MAT $\alpha$ yeast, namely the proteolytic maturation of $\alpha$-mating factor $(\alpha \mathrm{F})$ from its proprotein substrates. In various versions of this assay, the change in the mating activity of MATa yeast can be attributed to the loss of a critical component, such as Kex2p enzyme from the yeast's TGN (Sprague, 1991; Wilcox et al., 1992). To underscore this point, strains of MATa yeast that have null mutations in the KEX2 gene or that have been deleted (kex2A) can no longer mate (Leibowitz \& Wickner, 1976). Other seminal studies defined the processing proteases involved as being Kex2p, DPAP A, and another TGN resident proprotein protease, Kex1p (Bryant \& Stevens, 1997; Chen et al., 1997; Cooper \& Bussey, 1992; Fuller et al., 1988). All three must be co-localized to the TGN so that mature and biologically active 13 -amino acid $\alpha \mathrm{F}$ is properly processed from the much larger pro-mating-factor- $\alpha$ polypeptides that pass through this compartment. Properly processed biogenically active $\alpha \mathrm{F}$ peptides are then secreted to act as pheromones that attract MATa yeast for mating. In our studies we employed various mating assays to follow the localization and maintenance of Kex $2 p$ and the KFp chimeras in the yeast TGN. Qualitative analysis of mating by velveteen patch transfer clearly reveals in Fig. 5A that furin sorting signals are sufficient to localize the Kex2p enzymatic domain of the chimera to the TGN so that mating with a MATa strain (DC14) can occur. Quantitative mating results reveal that cells with normal vacuolar turnover (Pep4 ${ }^{+}$) cells (Fig. 5B and Table 2) expressing the chimera have a slightly lower 
degree of mating compared to those expressing Kex $2 p$, suggestive of a loss of the furin-targeted enzyme from the TGN to the cell surface. Interestingly, Pep4 ${ }^{-}$cells deficient in the vacuolar turnover of three key proteases (pep4 4 prc1 $\Delta$ prb1 $\Delta$ ) have higher levels of mating in both the Kex2p and chimera expressing cells (Table 2). This reflects the role of the vacuole in turnover and is consistent with results from the Fuller lab demonstrating increased levels of mating when Kex2p is expressed in Pep4 $4^{-}$cells. Even more intriguing is the observation from Table 2 that Pep4 $4^{-}$cells expressing KFp have higher levels of mating than Pep4 ${ }^{+}$cells expressing Kex2p. As Kex2p traffics by default to the vacuole where it is proteolytically turned-over in Pep4 ${ }^{+}$cells or tends to accumulate in Pep4- cells (Wilcox et al., 1992), it appears that in Pep4 $4^{-}$cells KF2p partitioned to a large cell-surface pool can be readily retrieved to endosomes by endocytosis and then by retrotransport to the yeast TGN. Thus a cell surface to endosome to TGN sorting pathway for furin, known to occur in animal cells (Thomas, 2002), appears to be paralleled by a yeast sorting pathway that recognizes furin sorting signals. Although, this is likely to involve a sorting process that does not involve AP-2 as this clathrin adaptin is not known to exist in yeast (Teuchert et al., 1999b).

\subsection{Mutagenesis of furin sorting signals disrupts retrotransport of KFp chimeras to the yeast TGN}

Previous studies demonstrated that the protein PACS-1 interacts with the AC of furin's cytoplasmic tail and that when the acidic residues or phosophorylatable serines are mutated it eliminates the ability of furin to be retrieved to the TGN (Takahashi et al., 1995; Voorhees et al., 1995; Wan et al., 1998). In the chimeras KF5p and KF6p we have recreated those mutations by substituting the acidic residue sequence $\mathrm{E}^{775} \mathrm{ED}$ with three alanines and by deleting the two phosphorylatable serines and the intervening aspartic acid at $\mathrm{S}^{773} \mathrm{DS}$, respectively (Fig. 2). As shown by our qualitative in Fig 5A, semiquantitative mating data in Fig. 5B and quantitative data in Table 2, little detectable mating activity could be elicited by either chimera with AC mutations (KF5p and KF6p). This data is consistent with the loss of the Kex2p enzyme from the TGN to forward compartments (endosomes and then the cell surface or vacuole) from which it can no longer be retrieved by a PACS-1-like mechanism.

A signal defined by the tyrosine in the YKGL motif of the furin cytoplasmic tail was previously shown to interact with clathrin adaptor protein AP-1 in human cells and to be necessary for its exit from the TGN to endosomes (Schäfer et al., 1995; Teuchert et al., 1999a, 1999b). To investigate whether this signal was being recognized in yeast we mutated the KF2 chimera $\left(\mathrm{Y}^{759} \rightarrow \mathrm{A}\right)$. Contrary to our expectations we observed that mating efficiency was slightly decreased in Pep $4^{+}$cells, indicating the exit of the $\mathrm{Y}^{759} \rightarrow \mathrm{A}$ chimera (KF3p) from the TGN (Table 2). Though it is an interesting observation, it is difficult to explain based on the parallel experiment in human cells where vesicular transport was impaired. Alternatively it is interesting to speculate that the YKGL signal may act more like the poorly defined TLS2 of Kex2p or DPAP A, because loss of such a signal would be consistent with the mating data for KF3p (Brickner \& Fuller, 1997; Bryant \& Stevens, 1997; Redding et al., 1996b).

\section{$3.5 \mathrm{KFp}$ Chimeras are poorly maintained in the yeast TGN in the absence of steady state expression}

The Fuller lab has used a sensitive version of the mating assay, called "onset of impotence" that measures the length of time needed for Kex2p to escape the TGN-endosome retrieval loop and to then be turned-over in the vacuole once its expression by a GAL1 promoter is repressed (Brickner \& Fuller, 1997; Redding et al., 1996; Wilcox et al., 1992). As the steady-state of the enzyme diminishes, the amount in the TGN decreases and so does mating efficiency. In these studies, the kinetics for the onset of impotence were carefully measured for native $\mathrm{Kex} 2 \mathrm{p}$ and $\mathrm{Kex} 2 \mathrm{p} \mathrm{Y}^{713} \rightarrow \mathrm{A}$, the latter of which has a disrupted TLS1 signal. Our Kex $2 \mathrm{p}$ control studies in Pep4 cells parallel those they previously obtained for onset of impotence. Moreover, we observed that the chimeras with intact AC motifs, KFJp and KF2p, have kinetics for the onset of impotence (Fig. 6C), that parallel those reported for the Kex2p Y ${ }^{713} \rightarrow$ A mutant (Wilcox et al., 1992). For Kex2p this mutation essentially eliminates the TLS1 signal needed for its retrieval from the PVC to the TGN, but leaves intact another, TLS2, that helps retain Kex2p in the TGN (Brickner \& Fuller, 1997, Redding et al,, 1996; Wilcox et al., 1992). Does this mean then that the chimera only experiences a single, transient passage through the compartment? Several lines of evidence argue against this interpretation, such as the findings concerned with the intracellular distribution of the chimera, but most notably because we have observed the near complete loss of mating when the $\mathrm{AC}$ of the furin portion of the chimera is mutated. Thus, we surmise that the intact AC motif in the furin portion of the KFp chimera seems be weakly involved in its retrotransport.

\section{Discussion}

While much is known about the trafficking of furin in animal cells and conversely the trafficking of Kex2p in yeast cells, until this study, little was known about how the sorting signals of the human furin protease might be recognized by the yeast's sorting machinery. Since it is the yeast's sorting machinery that seems to be recognizing part of the human protein's sorting signals, it is worth viewing furin's sorting within the context of 
Kex2p's well-known trafficking patterns. To this end, a model for the trafficking pathways of the KFp chimera in yeast has been proposed and is compared to those for Kex2p (Fig. 6).

\subsection{How does the KFp chimera traffic to the cell surface of yeast?}

A large fraction of the KFp chimera is demonstrated to be on the cell surface of yeast by our fractionation, intact cell, and immunofluorescence studies. This is somewhat surprising in that the vacuole is often viewed as the default pathway for membrane proteins in yeast (Roberts et al., 1992). The only apparent exit signal within the chimera for its transport to the cell surface of the yeast is the relatively long TMD of furin. If true, then its delivery might be by bulk-flow, perhaps, within lipid rafts being directed to the cell surface (Wieland et al., 1987; Raynor and Pelham, 1997, Lingwood, 2010). Alternatively it maybe a signaled process (Wang et al., 2006) involving an exomer-like coat (Fig 6B, path a1). At present it is not even possible to ascertain if the KFp chimera is accumulating at the cell surface by passing to it through an early endosomal compartment (Fig 6B, path a2) as the work of many furin investigators might suggest (Thomas, 2002). Moreover, the extent to which inefficient endocytosis may account for the large fraction of chimera at the cell surface is unknown as well.

\subsection{How does the KFp chimera undergo retrotransport to the yeast TGN?}

Although there is no apparent similarity in the cytoplasmic sorting signals for Kex $2 p$ and furin, there are many conserved structural and functional features that we have cited, including the high degree of sequence conservation in their N-terminal domains, shared enzymatic specificity, overall structural homology, and localization to the TGN. While an acidic cluster is not readily apparent in Kex2p, it is present in furin and many other retrotransported proteins (Bonifacino \& Rojas, 2006). The discovery of an acidic cluster in a variety of animal proteins prompted studies to finds a PACS-1-like molecule in yeast. In part these studies were undertaken because of the striking similarity of the yeast receptor Vps10p to the cation-independent mannose-6-phosphate receptor (CI-M6PR), which also contains an acidic cluster. Interestingly, in animal cells CI-M6PR is known to be retrieved from endosomes via redundant retrotransport pathways to the TGN. These redundant retrotransport pathways include the canonical retromer-based mechanism and two others involving Rab9/TIP47 and PACS-1 (Bonifacino \& Rojas, 2006; Krise et al., 2000; Seaman, 2005; Thomas, 2002). Another example is the retromer-based PVC to TGN retrieval of Kex2p that requires the sorting nexin Grd19p (Voos \& Stevens, 1998). Hettema et al. (2003) found that Snx4p, Snx41p and Snx42p will act as a complex required for the retrieval of the v-SNARE Snc1p from EE to the TGN. However, while Snx4p/41p/42p all have homology to animal sorting nexins, the retrieval of Snclp by these proteins is poorly understood and apparently a non-retromer-based mechanism. Might one or more of these mechanisms be involved in the retrotransport of KF2p? Given the high degree of conserved cellular machinery that typifies diverse eukaryotes, it was somewhat surprising that no structural homologs to PACS-1 have been found in yeast. Might there be a functional PACS-1 homolog that so poorly conserves the animal cell protein's structural features that it cannot be recognized in homology searches? Our data suggests a PACS-1-like molecule might exist in yeast, and so too, a hypothetical retrotransport mechanism.

\section{Conclusion}

By far, the most compelling finding in our study was the near complete loss of mating when the furin AC site of the KFp chimera had key acidic residues altered or serine residues deleted. While no structural ortholog of PACS-1 has been identified within the yeast genome, our preliminary mating data support a hypothesis that a functional homolog, if not a structural homolog of PACS-1, may exist in yeast. An unbiased or selective screen may serve to identify other genes linked to this hypothetical molecule. One approach might be to use a suppression of the onset of impotence (soi) screen similar to that used by the Fuller laboratory (Redding et al., 1996) to discover if there is a yeast sorting protein interacting with the AC motif in the KFp chimera. Another approach would involve studies of the KFp chimera in strains mutant for sorting proteins known to function in post-Golgi vesicular traffic, or to block the function of these sorting proteins, and thereby elucidate the pathways by which KFp traffics within yeast. These types of studies and others may prove key in determining anterograde (Fig. 6, a1 or a2) pathways and retrotransport pathways to the TGN via the EE or PVC (Fig. 6, r1 or r2, respectively).

\section{Acknowledgements}

The authors would like to acknowledge the intellectual contributions of J. Burchard and R. Fuller in the initial design of these studies and thank the following for their assistance with experiments: J. Burchard, A. Drake, T. Robertson, J. Arlt, S. Pucci, A. Montes de Oca, Z. Deckner, J. Miller, B. Rosen and S. Veamoi. 


\section{References}

Abazeed, M.E. \& Fuller, R.S. (2008). Yeast Golgi-localized, $\gamma$-Ear-containing, ADP-Ribosylation Factor-binding Proteins Are but Adaptor Protein-1 Is Not Required for Cell-free Transport of Membrane Proteins from the Trans-Golgi Network to the Prevacuolar Compartment. Mol. Biol. Cell, 19, 4826-4836.

Achstetter, T., Emter, O., Ehmann, C., \& Wolf, D.H. (1984). Proteolysis in eukaryotic cells. J. Biol. Chem., 259, 13334-13343.

Ballou, L., Hernandez, L.M., Alvarado, E., \& Ballou, C.E. (1990). Revision of the oligosaccharide structures of the yeast carboxypeptidase Y. PNAS U.S.A., 87, 3368-3372.

Boehm, M. \& Bonifacino, J.S. (2002). Genetic analyses of adaptin function from yeast to mammals. Gene, 286, 175-186.

Bonifacino, J.S. \& Benjamin G.S. (2004). The Mechanisms of Vesicle Budding and Fusion. Cell, 116, 153-166.

Bonifacino, J.S., \& Rojas, R. (2006). Retrograde transport from endosomes to the trans-Golgi network. Nature Reviews Molecular Cell Biology, 7, 568-579.

Bosshart, H., Humphrey, J., Deignan, E., Davidson, J., Drazba, J., Yuan, L., Oorschot, V., Peters, P.J., \& Bonifacino, J.S. (1994). The cytoplasmic domain mediates localization of furin to the trans-Golgi network en route to the endosomal/lysosomal system. J. Cell Biol., 126, 1157-1172.

Bowers, K. \& Stevens, T.H. (2005). Protein transport from the late Golgi to the vacuole in the yeast Saccharomyces cerevisiae. BBA-Mol. Cell Res., 1744, 438-454.

Brenner, C. \& Fuller, R.S. (1992). Structural and Enzymatic Characterization of a Purified ProhormoneProcessing Enzyme: Secreted, Soluble Kex2 Protease. PNAS U.S.A., 89, 922-926.

Brickner, J.H. \& Fuller, R.S. (1997). SOI1 Encodes a Novel, Conserved Protein That Promotes TGN-Endosomal Cycling of Kex2p and Other Membrane Proteins by Modulating the Function of Two TGN Localization Signals. J. Cell Biol., 139, 23-36.

Bryant, N.J. \& Stevens, T.H. (1997). Two Separate Signals Act Independently to Localize a Yeast Late Golgi Membrane Protein through a Combination of Retrieval and Retention. J Cell Biol., 136, 287-297.

Chen, P., Sapperstein, S.K., Choi, J.D., \& Michaelis, S. (1997). Biogenesis of the Saccharomyces cerevisiae Mating Pheromone a-Factor. J Cell Biol., 136, 251-269.

Cooper A. \& Bussey H. (1992). Yeast Kex1p is a Golgi-associated membrane protein: deletions in a cytoplasmic targeting domain result in mislocalization to the vacuolar membrane. J Cell Biol., 119, 1459-1468.

Crump C.M., Xiang Y., Thomas, L., Gu, F., Austin, C., Tooze S. A., \& Thomas G. (2001). PACS-1 binding to adaptors is required for acidic cluster motif-mediated protein traffic. EMBO J., 20(9), 2191-201.

Davis, T.N. \& Thorner, J. (1989). Vertebrate and yeast calmodulin, despite significant sequence divergence, are functionally interchangeable. PNAS U.S.A., 86, 7909-7913.

Dittié A.S., Thomas L., Thomas G., \& Tooze, S.A. (1997). Interaction of furin in immature secretory granules from neuroendocrine cells with the AP-1 adaptor complex is modulated by casein kinase II phosphorylation. EMBO J., 16(16), 4859-4870.

Ekena, K. \& Stevens, T.H. (1995). The Saccharomyces cerevisiae MVP1 Gene Interacts with VPS1 and is Required for Vacuolar Protein Sorting. Mol. Cell. Biol., 15(3), 1671-1678.

Franzusoff, A., Redding, K., Crosby, J., Fuller, R.S., \& Schekman, R. (1991). Localization of components involved in protein transport and processing through the yeast Golgi apparatus. J. Cell Biol, 112: 27-37.

Fuller, R.B., Brenner, C. Gluschankof, P., \& Wilcox, C.A. (1991). The yeast prohormone-processing Kex2 protease, an enzyme with specificity for paired basic residues. In Jörnall \& Höög (Eds.). Methods in Protein Sequence Analysis (pp. 205-214). Berlin, Birkhauser Verlag.

Fuller, R.S., Brake, A., \& Thorner, J. (1989a). Yeast Prohormone Processing Enzyme (KEX2 Gene Product) is a $\mathrm{Ca}^{+}$-Dependent Serine Protease. PNAS U.S.A., 86, 1434-1438.

Fuller, R.S., Brake, A.J., \& Thorner, J. (1989b). Intracellular targeting and structural conservation of a prohormone-processing endoprotease. Science, 246, 482-486.

Fuller, R. S., Sterne, R. E., \& Thorner, J. (1988). Enzymes Required for Yeast Prohormone Processing. Annu. Rev. Physiol., 50, 345-362. 
Ha, S., Bunch, J.T., Hama, H., DeWald, D.B., \& Nothwehr, S.F. (2001). A Novel Mechanism for Localizing Membrane Proteins to Yeast Trans-Golgi Network Requires Function of Synaptojanin-like Protein. Mol. Biol. Cell, 12, 3175-3190.

Hartwell, L.H. (1980). Mutants of Saccharomyces cerevisiae unresponsive to cell division control by polypeptide mating hormone. J. Cell Biol, 85, 811-822.

Hettema, E.H., Lewis, M.J., Black, M.W. \& Pelham, H.R. (2003). Retromer and the sorting nexins Snx4/41/42 mediate distinct retrieval pathways from yeast endosomes. EMBO J., 22, 548-557.

Johnston, M. \& Davis, R.W. (1984). Sequences that regulate the divergent GAL1-GAL10 promoter in Saccharomyces cerevisiae. Mol Cell Biol., 4, 1440-1448.

Julius, D., Brake, A., Blair, L., Kunisawa, R., \& Thorner, J. (1984). Isolation of the putative structural gene for the lysine-arginine-cleaving endopeptidase required for processing of yeast prepro-[alpha]-factor. Cell, 37: 1075-1089.

Krise J.P., Sincock P.M., Orsel J.G., \& Pfeffer S.R. (2000). Quantitative analysis of TIP47-receptor cytoplasmic domain interactions: implications for endosome-to-trans Golgi network trafficking. J. Biol Chem., 275, 25188-25193

Leibowitz, M.J., \& Wickner, R.B. (1976). A Chromosomal Gene Required for Killer Plasmid Expression, Mating, and Spore Maturation in Saccharomyces cerevisiae. PNAS U.S.A., 73, 2061-2065.

Lingwood D. \& Simons K. (2010). Lipid rafts as a membrane-organizing principle. Science, 327(5961),46-50.

Molloy, S.S., Anderson, E.D., Jean, F., \& Thomas, G. (1999). Bi-cycling the furin pathway: from TGN localization to pathogen activation and embryogenesis. Trends in Cell Biology, 9, 28-35.

Molloy S.S., Thomas, L., VanSlyke, J.K., Stenberg, P.E., Thomas, G. (1994). Intracellular trafficking and activation of the furin proprotein convertase: localization to the TGN and recycling from the cell surface. $E M B O$ $J ., 13,18-33$.

Payne, G.S., \& Schekman, R. (1989). Clathrin: a role in the intracellular retention of a Golgi membrane protein. Science, 245, 1358-1365.

Rayner J.C., \& Pelham H.R. (1997). Transmembrane domain-dependent sorting of proteins to the ER and plasma membrane in yeast. EMBO J., 16, 1832-1841.

Redding, K., Brickner, J.H., Marschall, L.G., Nichols, J.W., \& Fuller, R.S. (1996a). Allele-specific suppression of a defective trans-Golgi network (TGN) localization signal in Kex $2 p$ identifies three genes involved in localization of TGN transmembrane proteins. Mol. Cell. Biol., 16, 6208-6217.

Redding, K.S., Seeger, M., Payne, G. S., \& Fuller R. S. (1996b). The Effects of Clathrin Inactivation on Localization of Kex2 Protease Are Independent of the TGN Localization Signal in the Cytosolic Tail of Kex2p. Mol. Biol. Cell, 7, 1667-1677.

Rockwell, N.C., Krysan, D.J., Komiyama, T., \& Fuller, R.S. (2002). Precursor processing by Kex2/furin proteases. Chem. Rev., 102, 4525-4548.

Roberts, C.J., Nothwehr, S.F., \& Stevens, T.H. (1992). Membrane protein sorting in the yeast secretory pathway: evidence that the vacuole may be the default compartment. J. Cell Biol., 119, 69-83.

Rohn, W.M., Rouillé, Y, Waguri, S., Hoflack, B. (2000). Bi-directional trafficking between the trans-Golgi network and the endosomal/lysosomal system. J. Cell Sci., 113, 2093-101.

Sambrook, J., \& Russell, D.W. (2000). Molecular Cloning: A Laboratory Manual. (3rd ed.). Cold Spring Harbor, N.Y.: Cold Spring Harbor Laboratory Press.

Sato, K., Noda, Y., \& Yoda, K. (2009). Kei1: A Novel Subunit of Inositolphosphorylceramide Synthase, Essential for Its Enzyme Activity and Golgi Localization. Mol. Biol. Cell, 20, 4444-4457.

Schäfer, W., Stroh, A., Berghöfer, S., Seiler, J., Vey, M., Kruse, M.L., Kern, H.F., Klenk, H.D., \& Garten, W. (1995). Two independent targeting signals in the cytoplasmic domain determine trans-Golgi network localization and endosomal trafficking of the proprotein convertase furin. EMBO J., 14, 2424-2435

Seaman, M.N.J., McCaffery, J.M., \& Emr, S.D. (1998). A Membrane Coat Complex Essential for Endosome-to-Golgi Retrograde Transport in Yeast. J. Cell Biol., 142(3), 665-681.

Seaman, M.N.J. (2005). Recycle your receptors with retromer. Trends in Cell Biology, 15(2), 68-75. 
Sherman, F. (2002). Getting Started with Yeast. In C. Guthrie and G. Fink (Eds.) Methods of Enzymology Vol 351: Guide to Yeast Genetics and Molecular and Cell Biology, Part B (pp. 3-41). New York City: Academic Press, Elsevier.

Sipos, G. \& Fuller, R. S. (2002). Separation of Golgi and Endosomal Compartments, In C. Guthrie and G. Fink (Eds.). Methods of Enzymology Vol 352: Guide to Yeast Genetics and Molecular and Cell Biology, Part C (pp. 351-365). New York City: Academic Press, Elsevier.

Sipos, G., Brickner, J.H., Brace, E.J., Chen, L., Rambourg, A., Kepes, F., \& Fuller, R.S. (2004). Soi3p/Rav1p Functions at the Early Endosome to Regulate Endocytic Trafficking to the Vacuole and Localization of Trans-Golgi Network Transmembrane Proteins. Mol Biol Cell, 15, 3196-3209.

Sprague, G.F. (1991). Assay of Yeast Mating Reaction. In C. Guthrie and G. Fink (Eds.). Methods of Enzymology Vol 194: Guide to Yeast Genetics and Molecular and Cell Biology, Part A (pp. 77-92). New York City: Academic Press, Elsevier.

Takahashi, S., Nakagawa, T., Banno, T., Watanabe, T., Murakami, K., \& Nakayama, K. (1995). Localization of Furin to the trans-Golgi Network and Recycling from the Cell Surface Involves Ser and Tyr Residues within the Cytoplasmic Domain. J. Biol. Chem., 270, 28397-28401.

Teuchert M., Schäfer W., Berghöfer S., Hoflack B., Klenk H.D., \& Garten W. (1999a). Sorting of furin at the trans-Golgi network. Interaction of the cytoplasmic tail sorting signals with AP-1 Golgi-specific assembly proteins. J. Biol. Chem., 274, 8199-8207.

Teuchert, M., Berghöfer, S., Klenk, H.D., \& Garten W. (1999b). Recycling of furin from the plasma membrane. Functional importance of the cytoplasmic tail sorting signals and interaction with the AP-2 adaptor medium chain subunit. J. Biol. Chem., 274, 36781-36789.

Thomas, G. (2002). Furin at the cutting edge: from protein traffic to embryogenesis and disease. Nat. Rev. Mol. Cell. Biol., 3: 753-766.

van den Ouweland, A.M.W., van Duijnhoven, H.L.P., Keizer, G.D., Dorsser, L.C.J, Van de Ven, W.J.M. (1990). Sructural homology between the human fur gene product and the subtilisin-like protease encoded by yeast KEX2. Nucl Acids Res, 18, 664.

Verges, M. (2007). Retromer and sorting nexins in development. Frontiers in Bioscience, 12, 3825-3851.

Voorhees, P., Deignan, E., van Donselaar, E., Humphrey, J., Marks, M.S., Peters, P.J., \& Bonifacino, J.S. (1995). An acidic sequence within the cytoplasmic domain of furin functions as a determinant of trans-Golgi network localization and internalization from the cell surface. EMBO J., 14, 4961-4975.

Voos, W. \& Stevens, T. H. (1998). Retrieval of Resident Late-Golgi Membrane Proteins from the prevacuolar compartment of Saccharomyces cerevisiae is dependent on the function of Grd19p. J. Cell Biol., 140, 577-590.

Wan L., Molloy, S.S., Thomas, L., Liu, G., Xiang, Y., Rybak, S.L., \& Thomas, G. (1998). PACS-1 defines a novel gene family of cytosolic sorting proteins required for trans-Golgi network localization. Cell, 94, 205-216.

Wang, C-W., Hamamoto, S., Orci, L., \& Schekman, R. (2006). Exomer: a coat complex for transport of select membrane proteins from the trans-Golgi network to the plasma membrane in yeast. J. Cell Biol., 174(7), 973-83.

Wickner, R.B. \& Leibowitz, M.J. (1976). The Chromosomal Genes Required for Killing Expression in Killer Strains of Saccharomyces cerevisiae. Genetics, 82, 429-442.

Wilcox, C.A. \& Fuller, R.S. (1991). Posttranslational processing of the prohormone-cleaving Kex2 protease in the Saccharomyces cerevisiae secretory pathway. J. Cell Biol., 115, 297-307.

Wilcox, C.A., Redding, K., Wright, R., \& Fuller, R.S. (1992). Mutation of a tyrosine localization signal in the cytosolic tail of yeast Kex2 protease disrupts Golgi retention and results in default transport to the vacuole. Mol. Biol. Cell, 3, 1353-1371. 
Table 1. Site-specific indirect immunofluorescence recorded for Pep4 $4^{+}$cells expressing Kex2p and KFJp*

\begin{tabular}{|c|c|c|c|c|c|}
\hline & $\begin{array}{c}\text { \# Cells } \\
\text { Observed }\end{array}$ & $\begin{array}{c}\text { \% Fluorescently } \\
\text { Labeled Cells }\end{array}$ & Golgi Only & $\begin{array}{c}+ \text { Cell } \\
\text { Surface }\end{array}$ & Vacuole \\
\hline Kex2p & 71.5 & 47 & $95.8 \%$ & $2.8 \%$ & $1.4 \%$ \\
\hline KFJp & 375.5 & 32 & $59.7 \%$ & $39.4 \%$ & $0.9 \%$ \\
\hline No Enzyme & 215 & 1 & $0.0 \%$ & $0.0 \%$ & $0.7 \%$ \\
\hline
\end{tabular}

* Two investigators examined the photographs as described in the Materials and Methods.

$\dagger 97 \%$ of all cells showing cell surface fluorescence also had punctate staining typical of Golgi localization.

Table 2. Quantitative mating trials*

\begin{tabular}{|c|c|c|c|c|c|c|c|c|c|c|c|c|c|}
\hline & & \multicolumn{10}{|c|}{ Experiment Number } & \multirow{2}{*}{ Strain } \\
\cline { 3 - 14 } & Protease & 1 & 2 & 3 & 4 & 5 & 6 & 7 & 8 & 9 & 10 & 11 & Mean \pm SEM \\
\hline & Kex2p & 6.5 & 11.1 & 1.3 & 10.6 & 17 & 4.1 & 3.2 & 3.3 & 1.6 & - & - & $6.5 \pm 1.8$ \\
\hline & KF2p & 4.1 & 2.1 & 0.9 & 12.7 & 8.4 & 7.5 & - & 4.1 & 3.8 & - & - & $5.4 \pm 1.4$ \\
\hline Pep4 & KF3p & - & 1.3 & 0.4 & 6.6 & - & 3.4 & 1.7 & - & - & - & - & $2.4 \pm 1.1$ \\
\hline & KF5p & - & - & - & - & - & 0.02 & - & - & - & - & - & 0.02 \\
\hline & KF6p & - & - & - & - & - & 0.1 & - & - & - & - & - & 0.1 \\
\hline & & & & & & & & & & & & & \\
\hline & Kex2p & - & - & - & - & - & - & - & 7.5 & 4.6 & 10.6 & 10.3 & $8.2 \pm 1.4$ \\
\hline & KF2p & - & - & - & - & - & 8.9 & - & 6.9 & 8.2 & 14.6 & 7.4 & $9.2 \pm 1.4$ \\
\hline Pep4- & KF3p & - & - & - & - & - & 12.1 & - & - & - & 11.7 & 10.3 & $11.4 \pm 0.94$ \\
\hline & KF5p & - & - & - & - & - & 0.7 & - & - & - & 0 & 0 & $0.23 \pm 0.23$ \\
\hline & KF6p & - & - & - & - & - & 0.4 & - & - & - & 0 & 0 & $0.13 \pm 0.13$ \\
\hline
\end{tabular}

*Variation of method as previously described (Hartwell, 1980; Sprague, 1991). 200- $\mu$ l each of late log MATa and DC14 cells were combined and allowed to settle in microfuge tubes for $5 \mathrm{hrs}$ during which time mating could occur. After which tubes were briefly vortexed to suspend diploids and $100-\mu l$ of cells was spread onto plates, grown, and analyzed as described. 


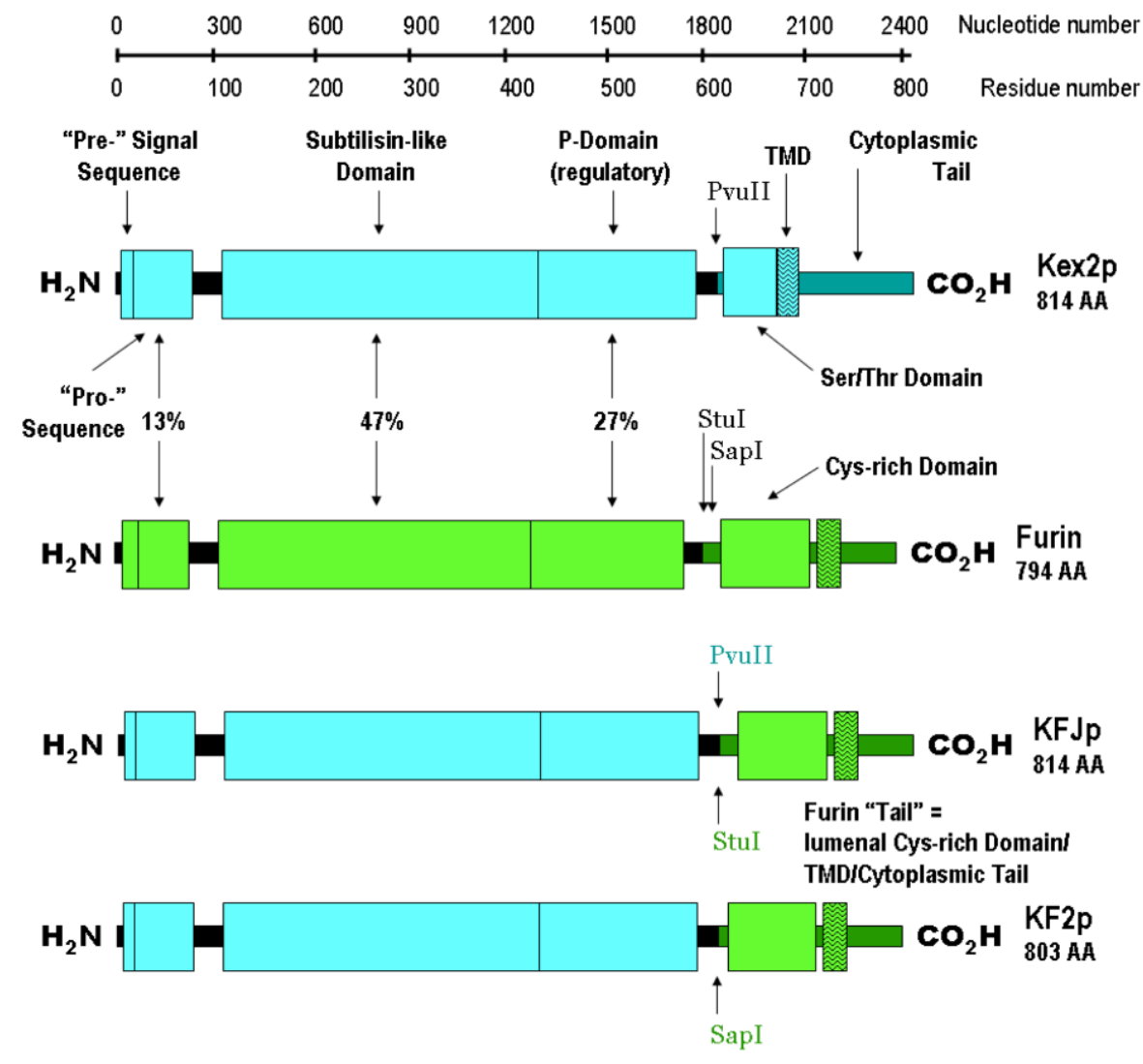

Figure 1. Schematic representations of Kex2p, furin and KFp chimeras. Glycosylation sites are not shown. Adapted from Fuller et al. (1991).

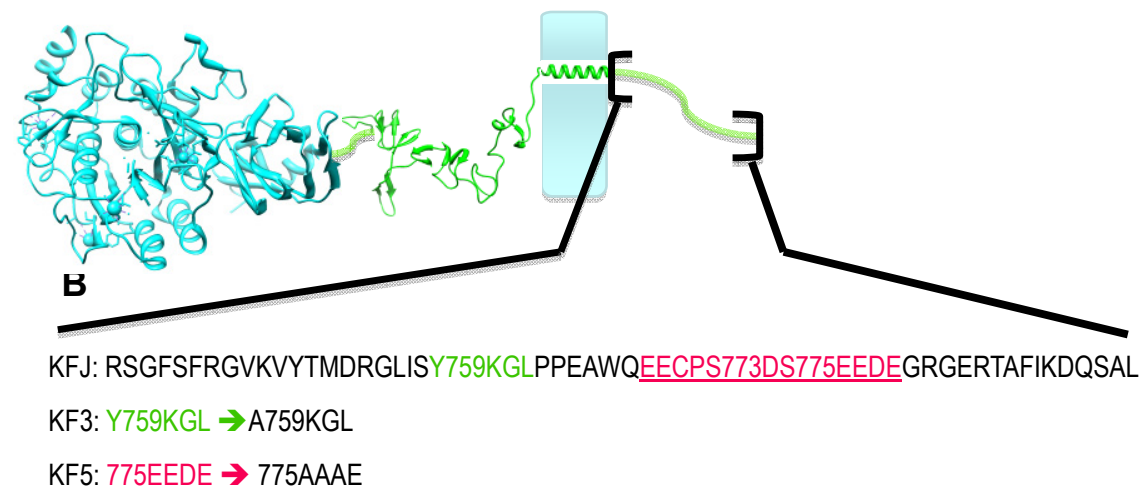

Figure 2. Representations of the KFp Chimera Structure. (A) KFJp and KF2p have an N-terminal Kex2p catalytic and P-domain (blue) fused in frame to furin's cysteine rich region, a single-pass transmembrane domain $\alpha$-helix, and cytosolic tail of unknown tertiary structure (green). X-ray diffraction studies have solved the catalytic and P domain of Kex2p and it is shown in blue. Structural homology of the lumenal Cys-rich spacer of furin with an epidermal growth factor receptor (EFGR) domain was used as a substitute for the Cys-rich domain for representation purposes. The combined image used RCSB files 1P8J, 1MOX, and 1R64 and was manipulated with the UCSF Chimera package. (B) The cytosolic tail sequence of furin (Van den Ouweland et al., 1989) is shown with highlighted residues that correlate to targets of site-directed mutagenesis used in this study. KFJp and KF2p have identical TMDs and cytosolic tails. They differ only in a few amino acids splicing the Kex2p enzyme to the furin $\mathrm{C}$-terminal domains. 
A

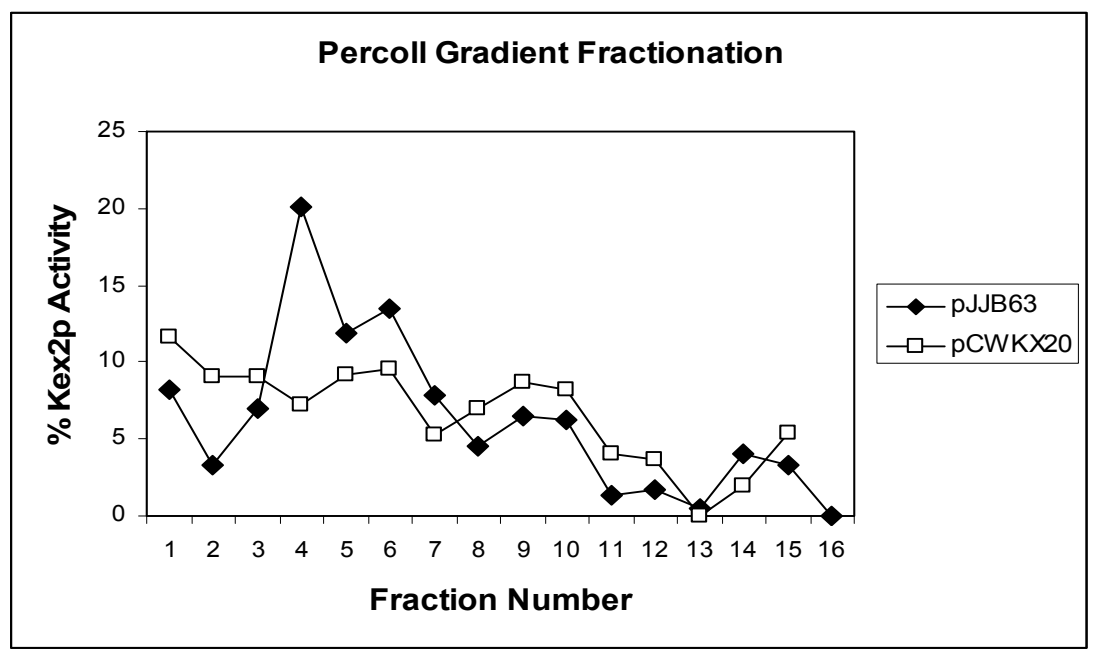

B

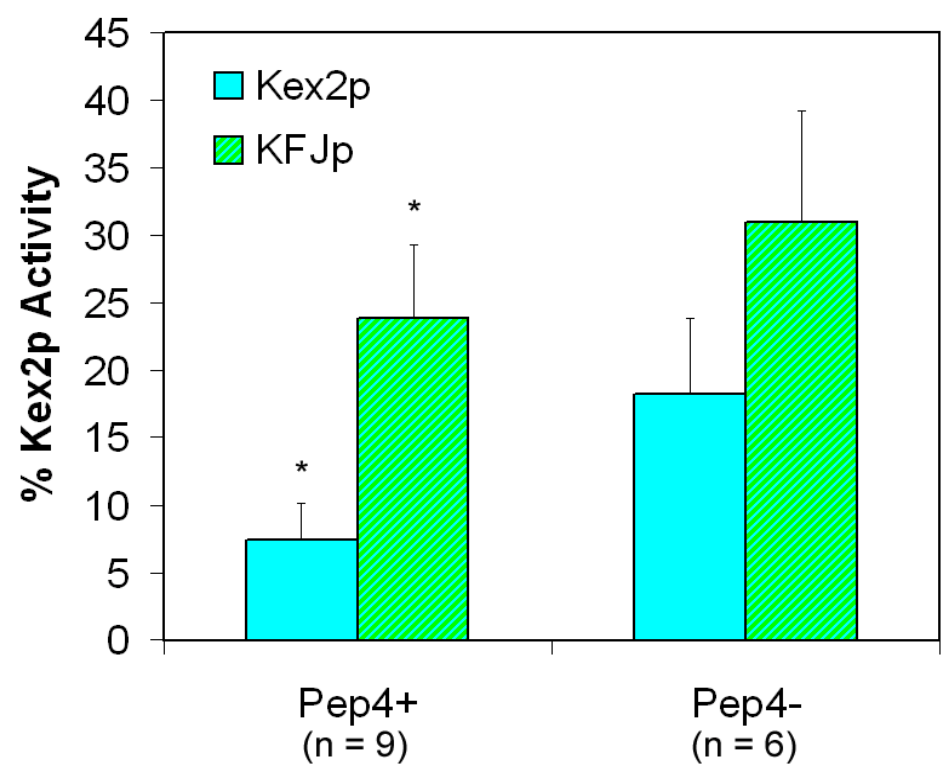

Figure 3. Kex2p activity present in subcellular fractions and on the surface of intact cells. (A) Sphero-plasted cells that were deficient in vacuolar proteases (Pep4 ${ }^{-}$cells) were dounced with a B pestle on ice, and loaded onto Percoll gradients in thin-wall polyallomer tubes and centrifuged for 3 hours at 21,000 rpm in a ss34 rotor using adaptors. Fractions were collected by a drip from the bottom of the centrifuge tube, thus the densest fraction was collected first. Gradient fractions were then tested for Kex2p-specific proteolytic activity as described. The relative activity of each fraction compared to the total activity observed across the gradient has been plotted. The legend indicates the plasmid used to express Kex2p and KFJp in the CB017 strain. pCWKX20 was the control plasmid for the expression of Kex2p and the plasmid designated pJJB63 is an earlier designation for pBM258-KFJp used to express the chimera KFJp. (B) Intact cell assay of Kex2p protease activity was compared to total activity present in the lysate of an equal volume of intact cells. Early log phase cells were induced by the addition of $2 \%$ galactose and incubated for 4 hours at $30^{\circ} \mathrm{C}$ prior to assay. During the assay, intact yeast cells were incubated for 60 minutes with gentle resuspension every 5 minutes. Pep $4^{+}$cell surface activity for KFJp was significantly different than Kex2p activity $(\mathrm{p}<0.04)$. Error bars represent standard error of mean. 

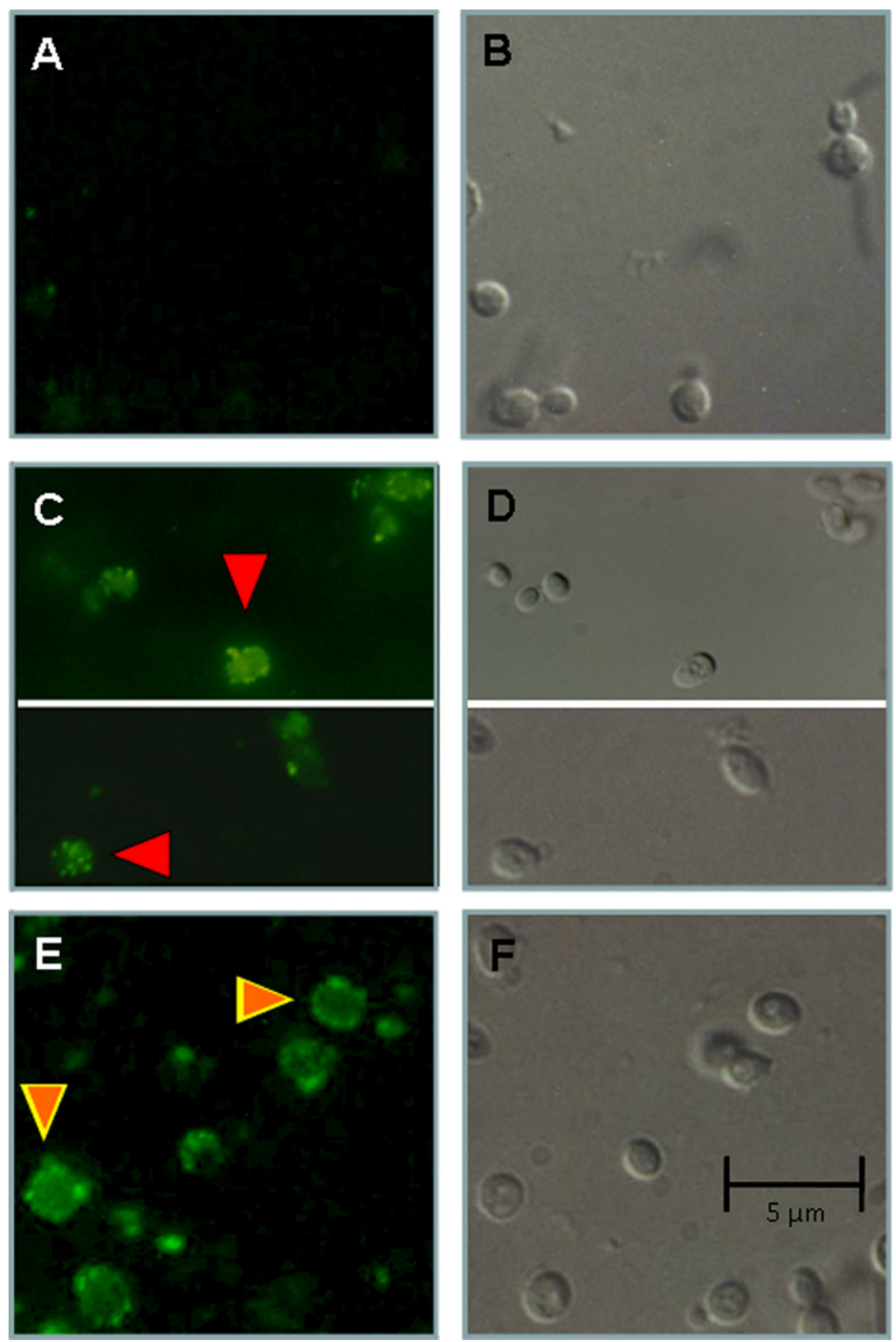

Figure 4. Immunofluorescence studies of the localization of the chimera by furin sorting signals. (A) The Pep4 kex $2 \Delta$ cells were grown to mid-log in $2 \%$ galactose SC-ura broth and conveyed the plasmids pBM258, pCWKX20, or pBM-KFJp that expressed (A) no enzyme (C) Kex2p or (E) KFJp. All cells were processed in parallel as described earlier. Companion DIC images are to the right (B, D, and F, respectively). (C) Clearly visible is the punctate staining of Golgi typical for Kex2p (red marker) and other resident Golgi proteins (Franzusoff et al., 1991). In (E) the faint halos of fluorescently labeled cell membrane along with some punctate labeling can be seen (orange markers). Bars in the DIC images indicate $5-\mu \mathrm{m}$. 

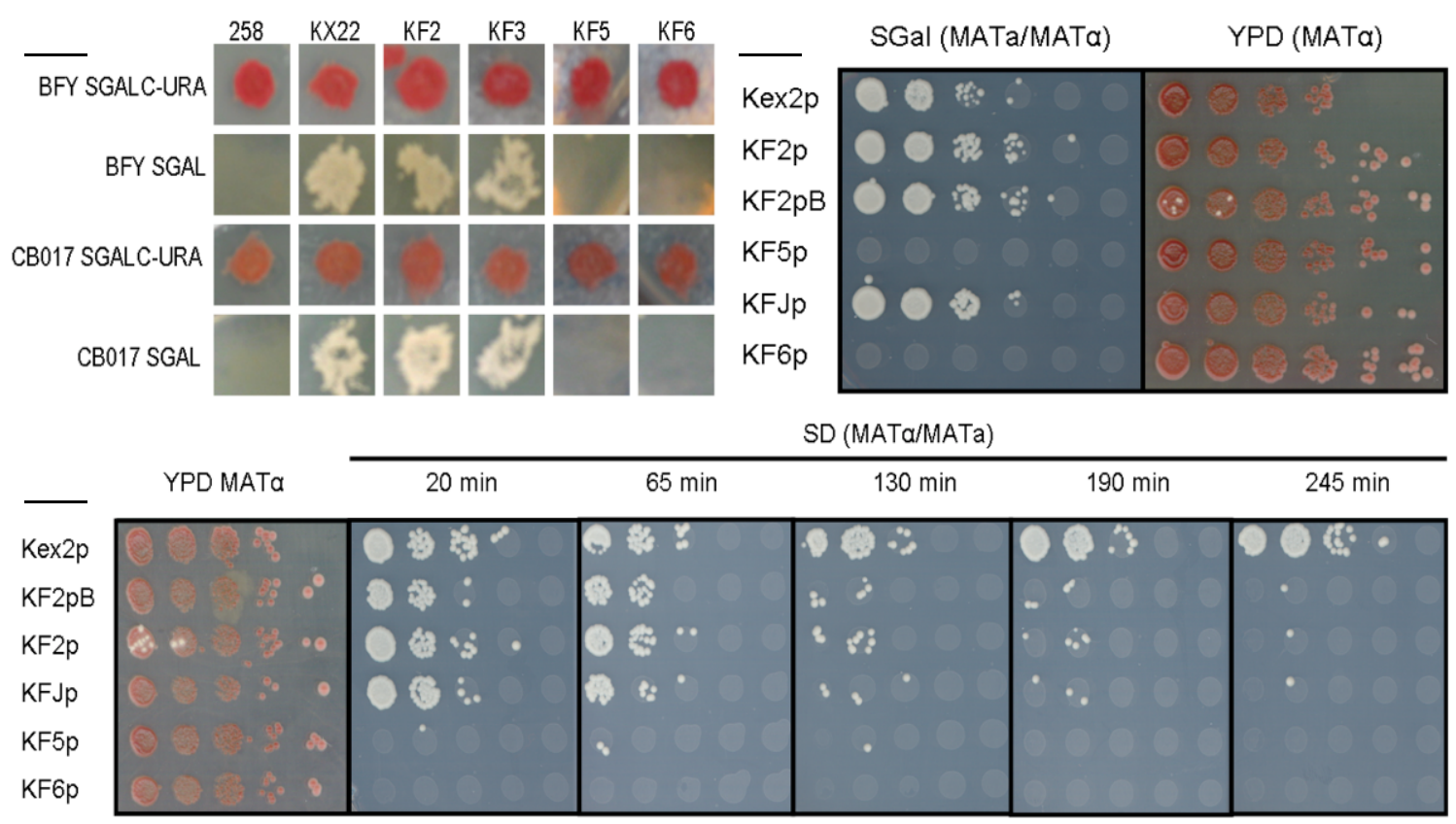

Figure 5. Qualitative and semi-quantitative comparisons of mating. (A) Initial patch mating of BFY106-4D $\left(\mathrm{Pep}^{+}\right)$and CB017 (Pep4 ${ }^{-}$) expressing Kex2p, KFJp or KF2p, or site-directed mutants of KF2p. (B) Plating of steady state Pep4 $4^{+}$cells with a replicating pin tool after 1:10 serial dilutions prior to mating (YPD) and after mating (SGal). (C) Onset of impotence in Pep4 ${ }^{+}$cells was determined by 1:10 dilution and incubation in SDC-ura broth for times shown, which was followed by plating as in B, except that SD plates were used instead of SGal. KF2pB and KF2p represent parallel cultures of BFY106-4D cells expressing pBM258-KF2.

A

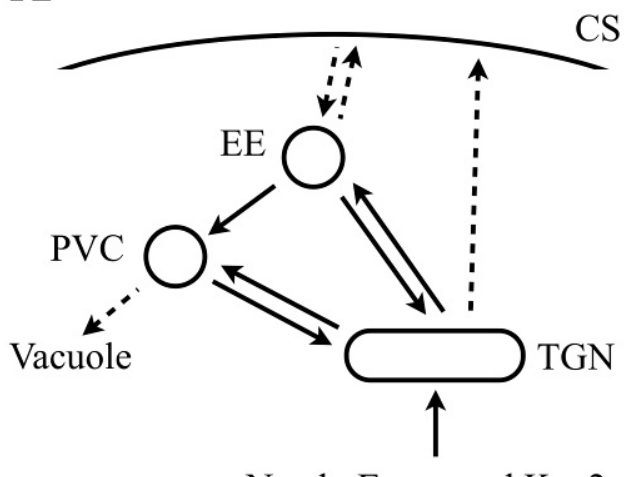

Newly Expressed Kex2p
B

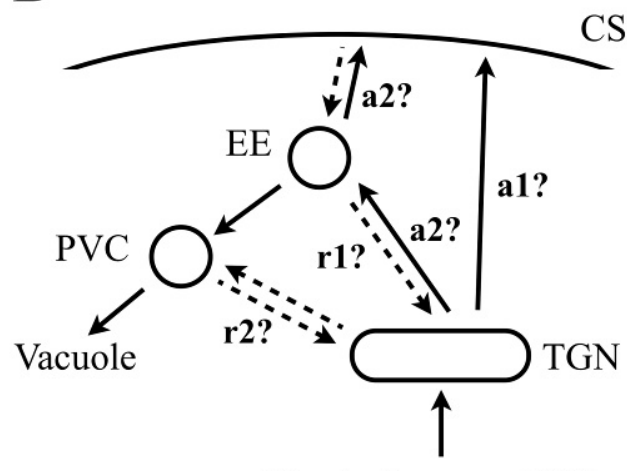

Newly Expressed KFp

Figure 6. Model for the vesicular trafficking of KFp in yeast. Solid arrows indicate principle pathways of transport. (A) Standard model described for the trafficking of Kex2p in yeast (Sipos et al., 2004). Note that strong retrotransport from both early endosome (EE) and the pre-vacuolar compartment (PVC) is indicated. (B) Proposed trafficking for KFp. Anterograde transport to the cell surface may occur through a TGN to cell surface (CS) pathway (a1) or a pathway through the $\mathrm{EE} \mathrm{(a2).} \mathrm{Also} \mathrm{indicated} \mathrm{is} \mathrm{the} \mathrm{relatively} \mathrm{weak} \mathrm{retrotransport} \mathrm{of} \mathrm{KFp}$ to TGN from either the EE (r1) or PVC (r2). 